\title{
Decay of Quantum Correlations in Atom Optics Billiards with Chaotic and Mixed Dynamics
}

\author{
M. F. Andersen, A. Kaplan, T. Grünzweig, and N. Davidson. \\ Department of Physics of Complex Systems, \\ Weizmann Institute of Science, \\ Rehovot 76100, Israel
}

(Dated: November 13, 2018)

\begin{abstract}
We perform echo spectroscopy on ultra cold atoms in atom optics billiards, to study their quantum dynamics. The detuning of the trapping laser is used to change the "perturbation", which causes a decay in the echo coherence. Two different regimes are observed: First, a perturbative regime in which the decay of echo coherence is non-monotonic and partial revivals of coherence are observed. These revivals are more pronounced in traps with mixed dynamics as compared to traps where the dynamics is fully chaotic. Next, for stronger perturbations, the decay becomes monotonic and independent of the strength of the perturbation. In this regime no clear distinction can be made between chaotic traps and traps with mixed dynamics.
\end{abstract}

The decoherence of superposition states, as they interact with the environment [1], is one of the main obstacles in realizing quantum information processing schemes [2]. Decoherence or dephasing represent loss of information, quantified by a decrease in the "fidelity", which typically measures the overlap between a desired state and the actual output of a real system.

In the field of classical chaos exponential sensitivity to initial conditions (non-vanishing Lyapunov exponent) plays a central role. However, the quantum analogue of evolving two initially slightly different wavefunctions in the same Hamiltonian yields that their overlap is constant in time, thereby not giving any information on the dynamics of the system. The fingerprints of the classically chaotic dynamics in the quantum regime (Quantum chaos) is a rich field of study [3]. One approach is to study the decay of the overlap between two initially identical wavefunctions evolved in slightly different Hamiltonians. The fidelity then denotes the overlap between a state evolved by a Hamiltonian $H_{\uparrow}$ with the same state evolved by a slightly perturbed Hamiltonian $H_{\downarrow}[4]$. The fidelity is in this context often denoted the Loschmidt Echo, since it is equivalent to the overlap between an initial state, and the same state evolved forward in time in $H_{\uparrow}$ and then backwards in time in $H_{\downarrow}[5]$. The decay of fidelity in chaotic systems and its dependence on several parameters, has been the topic of intense theoretical studies in recent years (see Ref. [4, 5, [6] and references therein). Nevertheless, experimental studies of chaotic systems are still lacking (mostly due to the difficulty of preparing highly-excited pure quantum states) and so are both theoretical and experimental investigations of systems with mixed dynamics. Of special interest in quantum dynamical studies are the "cross-over" regimes through which the system goes by changing its parameters from those values for which a quantum description is required, to those that allow classical models to be used. Understanding these regimes can shed light on the relation between classical and quantum properties of the system.

Ultra cold atoms have been used in the past to experimentally study both quantum and classical dynamics. Quantum dynamics have been studied in driven 1D systems, where a broad variety of phenomena such as dynamical localization [7], dynamical tunneling [8] and quantum accelerator modes [9] have been demonstrated. Classical dynamics has been studied in atom-optics billiards 10, 11], in which regular, chaotic and mixed dynamics were observed.

In this letter we use microwave (MW) "echo spectroscopy" 12 to experimentally measure the dephasing and quantum dynamics of ultra cold ${ }^{85} \mathrm{Rb}$ atoms trapped in atom-optics billiards, with underlying chaotic or mixed classical dynamics. Echo spectroscopy measures the overlap between two initially identical states evolved in slightly different Hamiltonians (and is therefore closely related to the fidelity [13]), but it overcomes the need for pure quantum states and allows the use of thermal ensembles for the study of quantum dynamics 12. We demonstrate that the decay of the echo coherence displays qualitative different behavior for different perturbation strengths. Two distinct regimes are observed. First, a "perturbative" regime where the decay depends on the perturbation strength and where partial coherence (wavepacket) revivals are observed even when the underlying classical dynamics is chaotic. This indicates that the partial revivals observed for a nearly harmonic trap 12 are in fact a generic feature of trapped atoms, as predicted in [13]. For stronger perturbations we observe a crossover to a regime where the decay of the echo coherence is independent of the perturbation strength. In this regime the decay is monotonic and no revivals are observed. For traps where the classical motion exhibits a mixed phase space (i.e. stable "islands" in a chaotic "sea") we observe more pronounced revivals in the perturbative regime, due to the periodic classical motion in the islands. However, a perturbation-independent regime exists also for these systems, and then the decay is 
essentially indistinguishable from that of traps with fully chaotic dynamics.

We use ${ }^{85} R b$ atoms in a coherent superposition of their two magnetic-insensitive hyperfine Zeemann ground states. These two levels, $\left|5 S_{1 / 2}, F=2, m_{F}=0\right\rangle$ denoted $|\downarrow\rangle$, and $\left|5 S_{1 / 2}, F=3, m_{F}=0\right\rangle$ denoted $|\uparrow\rangle$, are separated by the energy splitting $E_{H F}=\hbar \omega_{H F}$ with $\omega_{H F}=2 \pi \times 3.036 \mathrm{~s}^{-1}$. The atoms are trapped in a dipole potential formed by a linearly polarized laser close to the $5 S_{1 / 2} \rightarrow 5 P_{3 / 2}$ transition. The dipole potential is inversely proportional to the trap laser detuning $\Delta_{L}$, hence it is slightly different for atoms in $|\uparrow\rangle$ and $|\downarrow\rangle$. The external (center of mass) potential depends on the internal (spin) state, hence the internal and external degrees of freedom can not be separated, and the entire Hamiltonian (neglecting interactions between the atoms) is written as:

$$
\begin{aligned}
H & =H_{\downarrow}|\downarrow\rangle\left\langle\downarrow\left|+\left(H_{\uparrow}+E_{H F}\right)\right| \uparrow\right\rangle\langle\uparrow| \\
& =\left[\frac{p^{2}}{2 m}+V_{\downarrow}(\mathbf{x})\right]|\downarrow\rangle\left\langle\downarrow\left|+\left[\frac{p^{2}}{2 m}+V_{\uparrow}(\mathbf{x})+E_{H F}\right]\right| \uparrow\right\rangle
\end{aligned}
$$

where $V_{\downarrow}$ and $V_{\uparrow}$ are the external potentials for atoms in states $|\downarrow\rangle$ and $|\uparrow\rangle$, respectively. These potentials include the gravitational potential, equal for both states, and the dipole potential, which can be written as $V_{d, \downarrow}$ and $V_{d, \uparrow}=(1+\epsilon) V_{d, \downarrow}$, where $\epsilon \equiv \omega_{H F} / \Delta_{L}$ is the "perturbation strength" typically $10^{-3}-10^{-2}$ in our experiments [12].

The eigenenergies of this Hamiltonian consists of two manifolds (belonging to $|\downarrow\rangle$ and $|\uparrow\rangle$ ) separated in energy by $E_{H F}$. The atoms are initially prepared in their internal ground state $|\downarrow\rangle$ and their total wavefunction can be written as $\Psi=|\downarrow\rangle \otimes \psi$, where $\psi$ represents the center of mass part of their wavefunction. The echo sequence consists of three short and strong MW pulses, each of which changes the internal state of the atoms, while leaving the center of mass part of the wavefunction unchanged [12. First a $\pi / 2$-pulse puts the atoms into a coherent superposition of $|\downarrow\rangle$ and $|\uparrow\rangle$. After a time $\tau$ a $\pi$-pulse inverts the populations and after another time $\tau$ the atoms are irradiated by a second $\pi / 2$-pulse. The populations of $|\downarrow\rangle$ and $|\uparrow\rangle$ are then measured. If we start with an eigenstate $\left|n_{\downarrow}\right\rangle$ of $H_{\downarrow}$ then the population of $|\uparrow\rangle$ after the echo pulse sequence is 12$]: P_{\uparrow}=\frac{1}{2}\left[1-\operatorname{Re}\left(F_{\text {echo }}\right)\right]$, where $F_{\text {echo }}=\left\langle n_{\downarrow}\left|\mathrm{e}^{i H_{\downarrow} \tau} \mathrm{e}^{i H_{\uparrow} \tau} \mathrm{e}^{-i H_{\downarrow} \tau} \mathrm{e}^{-i H_{\uparrow} \tau}\right| n_{\downarrow}\right\rangle$ is denoted the "echo amplitude" (we omit $\hbar$ to simplify the equations). $F_{\text {echo }}=1$ indicates perfect coherence and yields $P_{\uparrow}=0$ and $F_{\text {echo }}=0$ yielding $P_{\uparrow}=0.5$ indicates complete loss of coherence. If $\epsilon=0$ then internal and center of mass motion degrees of freedom decouple and $F_{\text {echo }}=1$ for all times. When considering eigenstates the echo amplitude can be written as a time correlation function $F_{\text {echo }}=\mathrm{e}^{i \omega_{n, \downarrow} \tau}\left\langle\varphi_{n}(t=0) \mid \varphi_{n}(t=\tau)\right\rangle$ where $\left|\varphi_{n}(t=0)\right\rangle \equiv \mathrm{e}^{-i H_{\uparrow} \tau}\left|n_{\downarrow}\right\rangle$ and $\left|\varphi_{n}(t=\tau)\right\rangle \equiv$ $\mathrm{e}^{-i H_{\downarrow} \tau}\left|\varphi_{n}(t=0)\right\rangle$. Therefore, the decay of the echo coherence corresponds to a decay of quantum correlations
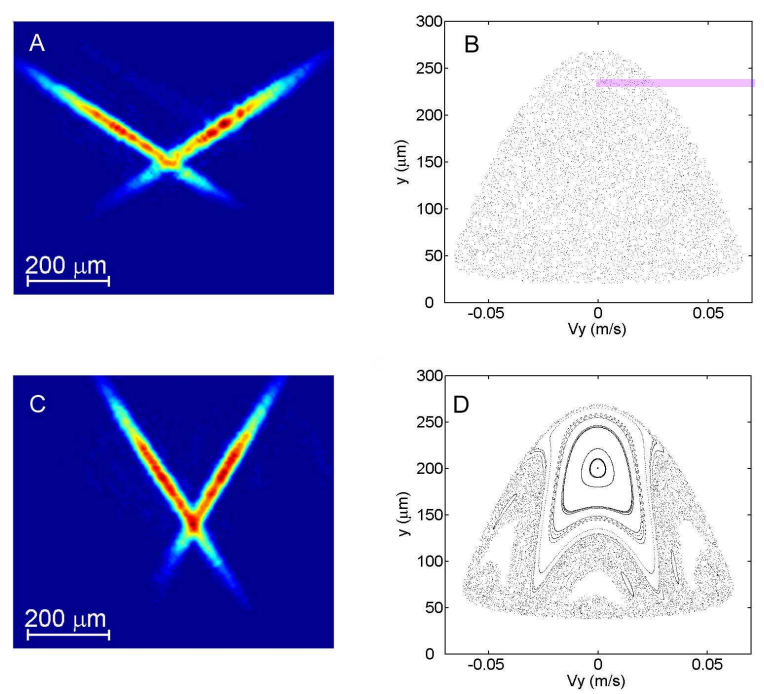

FIG. 1: (Color online) CCD images of edge billiards with $\alpha=52.5^{\circ}(\mathrm{A})$ and $\alpha=31^{\circ}(\mathrm{C})$, used in our experiments and their corresponding calculated Poincaré surface of sections, respectively, indicating chaotic dynamics (B) and mixed dynamics with large islands of stability (D).

due to dynamics in the trap.

In our experiment ${ }^{85} R b$ atoms are loaded into a far off resonance optical trap, cooled to a temperature of 20 $\mu K$, and optically pumped into the $\mathrm{F}=2$ hyperfine state. By changing the detuning of the trap laser, and simultaneously adjusting its power, the perturbation strength is controlled. After the MW echo pulses $P_{\uparrow}$, the population of state $|\uparrow\rangle$, is measured using fluorescence detection, and the signal is normalized to $P_{\uparrow}$ after a short $\pi$-pulse.

The trap is a light-sheet wedge billiard, made from two crossed blue detuned light sheets defining the billiard walls and where gravity confines the atoms in the vertical direction [14]. The light sheets have $\left(1 / e^{2}\right)$ dimensions of $20 \times 250 \mu \mathrm{m}$, and by the use of cylindrical lenses mounted on rotational stages, the wedge angle are adjusted in order to control the classical dynamics. The very elongated shape of the trap allows us to consider only the transverse motion and neglect the longitudinal one, which has a timescale much longer than the experiment time. The temperature of the atoms is much larger than the mean level spacing in the trap, and the atoms typically occupy many (up to $\sim 10^{8}$ ) states in the trap. The measured echo signal is the ensemble average of all of them.

The structure of phase space in the wedge billiard can be tuned from stability to chaos by varying the vertex half-angle, $\alpha$ [15]. For $\alpha<45^{\circ}$ phase space is mixed, and the size of the stable islands oscillates as a function of $\alpha$, a behavior dubbed "breathing chaos". For $\alpha>45^{\circ}$ the system is fully chaotic. Figures $1 \mathrm{~B}$ and 1D present the Poincaré surface of section for the two wedge billiards of Fig. 1A and 1C, respectively, used in our experiments, 
calculated by numerical integration of the classical trajectories in the measured billiard potentials. As seen, the classical dynamics is indeed almost fully chaotic and mixed for $\alpha=52.5^{\circ}$ and $\alpha=31^{\circ}$, respectively, as predicted [15] and previously measured [10].

As shown in Ref. 13], the echo amplitude for small perturbations can be written as:

$$
\begin{gathered}
F_{E C H}\left(\left|n_{\downarrow}\right\rangle, \tau\right) \simeq 4 \sum_{m \neq n} \exp \left(-i \omega_{m} \tau\right)\left|\left\langle m_{\uparrow} \mid n_{\downarrow}\right\rangle\right|^{2}- \\
\sum_{n \neq m} \exp \left(-2 i \omega_{m} \tau\right)\left|\left\langle m_{\uparrow} \mid n_{\downarrow}\right\rangle\right|^{2}+\left|\left\langle n_{\uparrow} \mid n_{\downarrow}\right\rangle\right|^{6},
\end{gathered}
$$

This means that the perturbative echo signal for a monoenergetic ensemble of atoms can be expressed as a function of the local density of states (LDOS). The LDOS denotes the local average of the absolute value squared of the matrix elements of the transformation matrix from eigenstates of $H_{\downarrow}$ to eigenstates of $H_{\uparrow}$. Formally, it is simply $\left|\left\langle m_{\uparrow} \mid n_{\downarrow}\right\rangle\right|^{2}$ as a function of $m_{\uparrow}-n_{\downarrow}$ averaged over a ensemble of neighboring $n_{\downarrow}$ with approximately the same energy. The width over which the LDOS is nonvanishing is denoted the "bandwidth", and if it is large, we expect a rapid decay of the echo coherence. In the perturbative regime the LDOS displays system specific features, despite the fact that the underlying classical dynamics is chaotic. In particular it is evident from the semiclassical calculations of [13, 16] that for atom optics billiards, where the inherent perturbation is localized on the billiard walls, the LDOS will have pronounced peaks for $m_{\uparrow}-n_{\downarrow}$ corresponding to $E_{n}-E_{m}=h / \tau_{b l}$, where $\tau_{b l}$ is the typical time between encounters with the wall $(\simeq 15$ $\mathrm{ms}$ in our experiments). This means that the echo amplitude will show partial revivals for $\tau=\tau_{b l}[13]$.

In Fig. 2 the decay of the echo signal for different perturbations is presented for a wedge with $\alpha=52.5^{\circ}$ (see Fig. 1A) where the dynamics is almost fully chaotic, as shown in Fig. 13. For small perturbations a nonmonotonic decay is seen with a partial revival of correlations for $\tau \simeq \tau_{b l}$ as predicted in [13]. The revivals are seen despite the fact that due to the "high" temperature a large band of energies is occupied in the trap. Partial revivals of correlations in traps where the dynamics is separable are generally expected at time scales equivalent to the 1D level spacing, but their surprising observation here, for a trap with chaotic motion, demonstrates that they are a far more widespread phenomena.

For larger perturbations a crossover to a regime where the decay is monotonic is observed. In this regime the decay is independent of perturbation strength. This is evident from Fig. 3 in which $P_{\uparrow}(\tau=0.0025 s)$ is plotted as a function of perturbation strength. It is seen that $P_{\uparrow}$ initially grows with perturbation strength but for $\epsilon>0.004, P_{\uparrow}$ is roughly constant. The perturbation independent regime of the echo decay is associated with the nonuniversal regime of [16], where the LDOS was

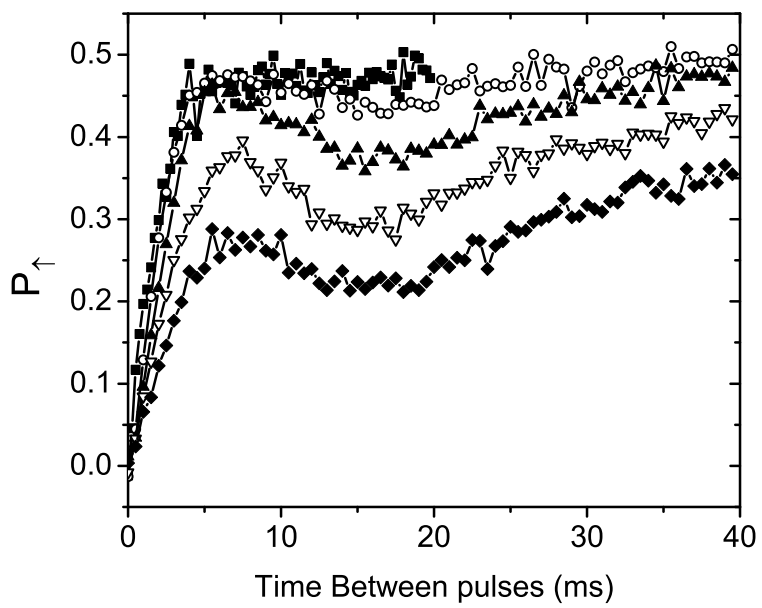

FIG. 2: Echo signal for a light sheet wedge with chaotic classical dynamics, for different perturbation strengths (trap laser wavelength tuned from $\lambda=775.9$ to $\lambda=779.7)$. $\epsilon=1.44 \times 10^{-3}, \nabla: \epsilon=1.90 \times 10^{-3}, \mathbf{\Delta}: \epsilon=2.43 \times 10^{-3}, \circ:$ $\epsilon=3.80 \times 10^{-3}$ and $\mathbf{\square}: \epsilon=1.52 \times 10^{-2}$. For small perturbations a nonmonotonic decay with revivals around $\tau=\tau_{b l}$ is seen, whereas for large ones a monotonic decay is observed.

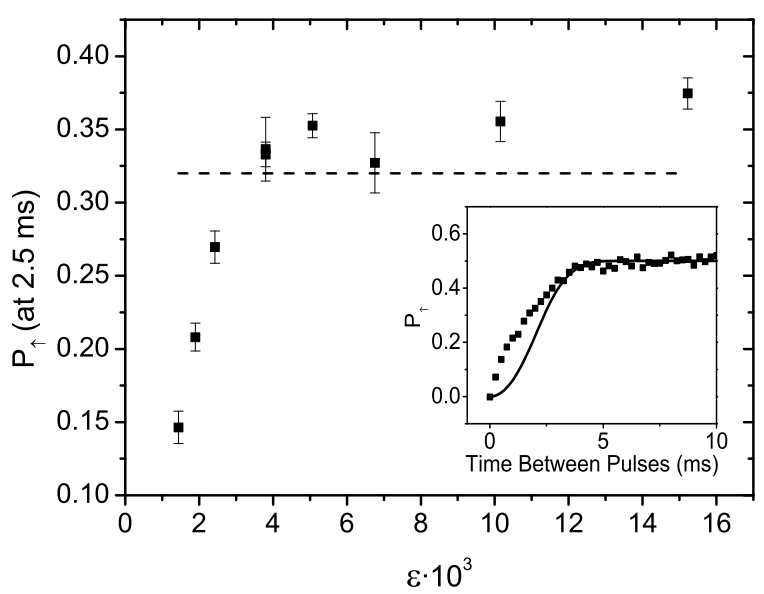

FIG. 3: Echo signal for a time between pulses of $2.5 \mathrm{~ms}$, as a function of perturbation strength. For a small perturbation the decay depends strongly on the perturbation strength, whereas for $\epsilon>4 \times 10^{-3}$ the decay is almost independent of perturbation strength. Dashed line: $P_{\uparrow}($ at $2.5 \mathrm{~ms})$ predicted by the simple classical model described in the text. Inset: Solid line: $P_{\uparrow}$ calculated by classical model. $\mathbf{\square}: P_{\uparrow}$ measured for a perturbation of $\epsilon=1.52 \times 10^{-2}$.

found to be perturbation independent. In this regime the perturbation is large enough so the overlap of equivalent eigenstates is small, and this indicates that the effects of quantization of the trap levels should not play a role and a classical description might be possible. Since the echo amplitude can be viewed as a propagator, and in our system the thermal de-Broglie wavelength is much smaller than the billiard's dimensions, it is possible to use a semiclassical propagator [3]. It is then seen that the classical 


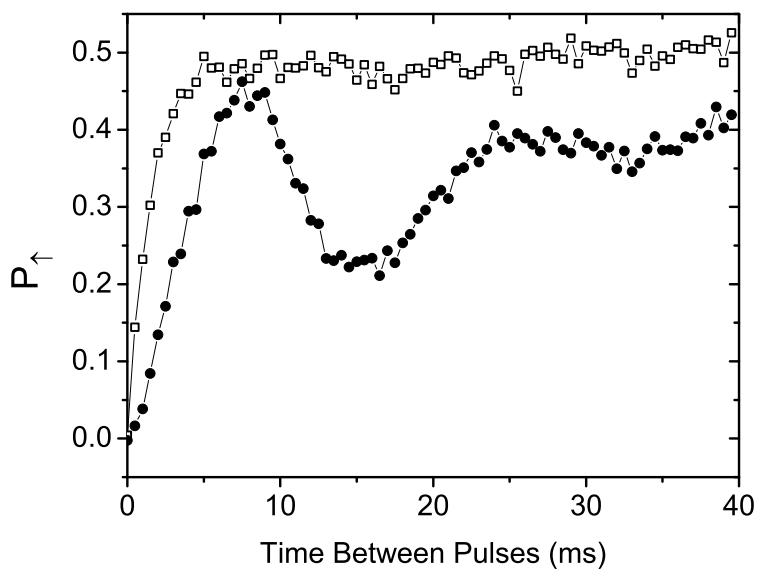

FIG. 4: Echo signal for a light sheet wedge where the classical phase-space is mixed. $\bullet: \epsilon=1.44 \times 10^{-3}$. $\square: \epsilon=1.52 \times 10^{-2}$. For the small perturbation a revival that is more pronounced than the one in the trap with classical chaotic dynamics is observed, whereas for large perturbations the decay can not be distinguished from the decay in a trap with chaotic dynamics.

trajectories contributing to the ensemble average of the echo amplitude, are those that after evolving forward in time in $H_{\uparrow}$ and $H_{\downarrow}$, and then backwards in time in $H_{\uparrow}$ and $H_{\downarrow}$, return to the vicinity of their initial position. These trajectories we divide into two types: those that during this propagation have hit the wall, and those that did not. Since $H_{\uparrow}$ and $H_{\downarrow}$ are highly different mainly in the vicinity of the wall, then the action integral along the first type of trajectories will yield a very large phase and the contribution from these trajectories to the ensemble average of the echo amplitude will average out. However since the second type of trajectories does not feel the difference between the potentials it will retrace its forward propagation backwards in time causing the action integral to vanish. These trajectories will give a perfect contribution. Therefore the echo amplitude in this regime simply measures the probability that the particles have not yet hit the wall, and this is a classical quantity. The dashed line in Fig. 3 describes the classical estimation of $P_{\uparrow}(\tau=0.0025 s)$ calculated assuming an idealized hard wall wedge populated with a thermal ensemble of atoms at a temperature of $20 \mu K$, clipped at an energy equal to the depth of the trap. In the inset of Fig. 3 the classical calculation is shown together with the measured echo decay for a perturbation of $\epsilon=1.5 \times 10^{-2}$, and reasonable agreement is seen despite the extreme simplicity of the above model.

Next we consider billiards with mixed dynamics. The semiclassical perturbative calculations of 13, 16] indicate that classical periodic motion as in elliptic islands will lead to narrow peaks in the LDOS, thereby yielding more pronounced revivals compared with the chaotic motion discussed above. This is observed in Fig. 4 where the decay of the echo signal is seen for the mixed- dynamics billiard with $\alpha=31^{\circ}$, shown in Fig. 11 The revival for small perturbation is deeper than for the chaotic motion of Fig. 2] and the reminiscence of a second revival for $\tau \simeq 2 \tau_{b l}$ can be seen indicating that the peak in the LDOS is narrower. Since the revivals are more pronounced in the case of mixed phase space they "survive" to slightly larger perturbation strength. However, in the perturbation-independent regime also shown in Fig. 4 the decay is essentially indistinguishable from that of the chaotic billiard, in agreement with the classical model given above.

In summary, we studied the decay of quantum correlations in atom optics billiards in which the classical dynamics is chaotic or mixed. We observed two distinct regimes for the perturbation strength in which the decay was qualitatively different. In the perturbative regime the decay was non-monotonic, with revivals at times corresponding to the typical time between bounces from the wall. The revivals were more pronounced in traps with mixed phase-space as compared with traps where the dynamics is almost fully chaotic. In the perturbative regime the decay rate increased with perturbation strength. However, in the perturbation independent regime the decay was monotonic and independent of perturbation strength. No clear distinction between the decay in traps with mixed and chaotic dynamics could be made in the perturbation independent regime. In this regime the echo amplitude measures the classical probability that an atom have not yet hit the wall.

The authors gratefully acknowledge discussions with U. Smilansky, D. Cohen, T. Kottos and K. Molmer. This work was supported in part by the Israel Science Foundation and the Minerva Foundation.

[1] W.H. Zurek., Rev. Mod. Phys. 75, 715 (2003).

[2] D. P. DiVincenzo, Science 270, 255 (1995).

[3] M. C. Gutzwiller. Chaos in Classical and Quantum Mechanics, Springer-Verlag, New York (1990).

[4] A. Peres, Phys. Rev. A 30, 1610 (1984).

[5] R. A. Jalabert and H. M. Pastawski, Phys. Rev. Lett., 86, 2490 (2001).

[6] J. Emerson, Y. S. Weinstein, S. Lloyd, and D. G. Cory, Phys. Rev. Lett. 89, 284102 (2002); Ph. Jacquod, I. Adagideli, and C. W. J. Beenakker, Phys. Rev. Lett. 89, 154103 (2002); F.M. Cucchietti, H.M. Pastawski, and D. A. Wisniacki, Phys. Rev. E 65, 045206(R) (2002); T. Prosen and M. Znidaric, New J. Phys. 5109 (2003); R. Sankaranarayanan, and A. Lakshminarayan, Phys. Rev E 68036216 (2003), M. Hiller, T. Kottos, D. Cohen, and T. Geisel., Phys. Rev. Lett. 92, 010402 (2004).

[7] M. Raizen, Adv. At. Mol. Opt. Phys. 41, 43 (1999).

[8] D. A. Steck, W. H. Oskay, and M. G. Raizen, Science 293, 274 (2001); W. K. Hensinger et. al., Nature 412, 52 (2001).

[9] M. Oberthaler et. al., Phys. Rev. Lett. 83, 4447 (1999); S. Schlunk, M.B. d'Arcy, S. A. Gardiner, and G. S. 
Summy, Phys. Rev. Lett. 90, 124102 (2003).

[10] V. Milner, J. L. Hanssen, W. C. Campbell and M. G. Raizen, Phys. Rev. Lett. 86, 1514 (2001).

[11] N. Friedman, A. Kaplan, D. Carasso and N. Davidson, Phys. Rev. Lett. 86, 1518 (2001); A. Kaplan, N. Friedman, M. Andersen, and N. Davidson, Phys. Rev. Lett. 87, 274101 (2001).

[12] M. F. Andersen, A. Kaplan and N. Davidson, Phys. Rev. Lett. 90, 023001 (2003).
[13] M. F. Andersen, T. Grunzweig, A. Kaplan, and N. Davidson, Phys. Rev. A, in press (2004).

[14] N. Davidson et. al., Phys. Rev. Lett. 74, 1311 (1995).

[15] H. E. Lehtihet and B. N. Miller, Physica (Amsterdam) 21D, 93 (1986).

[16] D. Cohen and T. Kottos, Phys. Rev. E, 63, 036203 (2001); D. Cohen, A. Barnett and E. J. Heller, Phys. Rev. E, 63, 046207 (2001). 